\title{
Efficacy of Vein Mapping in Lymphaticovenular Anastomosis
}

\author{
Yukiko Kuramoto, MD*; Kenta Tanakura, MD; Masayuki Sawaizumi, MD \\ The Cancer Institute Hospital, Japanese Foundation for Cancer Research, Koto, Tokyo, Japan
}

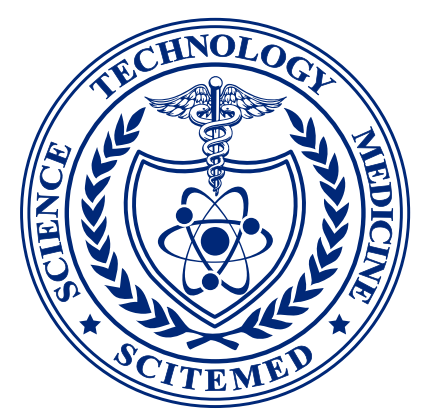

\begin{abstract}
For successful lymphaticovenular anastomosis (LVA), it is important that both lymphatics and veins should be placed within one small incision. We report a case of vein mapping using VueTek's Veinsite ${ }^{\mathrm{TM}}$, a non-contact vein visualization system. A 68-year-old Japanese woman had noted worsening lymphedema in both her lower limbs for five years. Seven years ago, she underwent total abdominal hysterectomy, bilateral salpingo-oophorectomy, pelvic lymphadenectomy, para-aortic lymphadenectomy, and omentectomy. Despite the constant and strong compressive therapy, edema gradually progressed. The ICG fluorescent test showed several intact lymph-duct flows to the bilateral lower thigh. The bilateral thigh showed severe abnormal images of dermal backflow. Veinsite ${ }^{\mathrm{TM}}$ was used to identify and map subcutaneous venules before surgery. The points of intersections between lymph-duct and venules were eleven in the right lower thigh and nine in the left lower thigh. We performed LVAs under local anesthesia (on the left leg: two incisions and three LVAs; on the right leg: three incisions and four LVAs). At all the incision sites, we found lymphatics and venules at suitably adjacent places for LVA. The lymphedema showed signs of improvement at 6 months post-surgery and the pain in the lower extremities also disappeared. Veinsite ${ }^{T M}$ is a new non-contact and non-invasive vascular imaging device that uses near-infrared light. It can detect reduced hemoglobin at about $0.7 \mathrm{~cm}$ depth from the surface of the skin. This device shows veins easily, quickly, and safely by displaying their precise positions on the monitor. Its description range is wide and we can use it in hands-free mode. In the case described here, seven LVAs were done successfully at five incisions. The most difficult part of the LVA procedure is in determining where to place the skin incision. Vein mapping can provide additional information to determine LVA site preoperatively, thus increasing the success rate of LVA.
\end{abstract}

\section{Introduction}

For successful lymphaticovenular anastomosis (LVA), it is important that both lymphatics and veins should be placed within one small incision. Lymphatics can be mapped with Indocyanine green (ICG) fluorescent lymphography [1,2], using specific infrared camera like Photo Dynamic Eye (PDE, Hamamatsu Photonics, Japan). However, we cannot know where the suitable venules exist before surgery. Only large venous vessels are detectable by PDE slightly. So, we sometimes encounter cases where the detection of a suitable venule becomes the most time-consuming process during the surgery. Recently, some types of vein visualization system have been developed. In this article, we report a case of vein mapping using VueTek's Veinsite ${ }^{\mathrm{TM}}$, a non-contact vein visualization system (Figure $1 \mathrm{~A}, 1 \mathrm{~B}$, and $1 \mathrm{C})$.

\section{Case Report}

A 68-year-old Japanese woman had worsening lymphedema in both her lower limbs over the last five years. Seven years ago, she underwent total abdominal hysterectomy, bilateral salpingo-oophorectomy, pelvic lymphadenectomy, para-aortic lymphadenectomy, and omentectomy. Despite the constant use of strong compressive therapy by elastic stocking during the last five years, her lymphedema gradually progressed and she felt pain in both legs. The ICG fluorescent test showed several intact lymphatics from her dorsum pedis to lower thigh in both legs. Dermal backflow sign was visible on both thighs. As a preoperative preparation, first, the lymphatic vessels were detected and mapped by red lines using PDE. Then, Veinsite ${ }^{\mathrm{TM}}$ was used to identify and map subcutaneous veins and venules by black lines just around the lymphatics. The width of the lines could show the thickness of the detected veins and venules. The points of intersections between lymphatics and venules were eleven on the right side and nine on the left side (Figure 1D). We performed LVAs under local anesthesia (on the left leg: two for ankle and one for thigh; on the right leg: one for ankle, one for thigh, and two for knee). At all the incision sites, we found lymphatics and venules at suitably adjacent places for LVA. As a result, venules were detected approximately $0.5 \mathrm{~mm}$ in thickness. Compression therapy was resumed postoperatively. The lymphedema showed signs of improvement at 6 months post-surgery and the pain in the lower extremities also disappeared. Lower Extremity Lymphedema (LEL) index [3] improved from 247 to 220 in the right lower limb, and 281 to 237 in the left lower limb (Figure 1E and 1F).

\section{Discussion}

Non-contact vascular imaging devices were developed to facilitate the puncture of veins in cases where vein detection was difficult, especially in babies and infants. Projection type vein visualization devices could make vein visible, but had difficulty in identifying venules $[4,5]$. Also, the device is handled mainly by hands. Veinsite ${ }^{\mathrm{TM}}$ is a new non-contact, non-invasive vascular imaging device that uses near-infrared light. It can detect reduced hemoglobin at about $0.7 \mathrm{~cm}$ depth beneath the surface of the skin. This device shows veins easily, quickly, and safely by displaying their precise positions on the monitor. This device is head-mounted; so, the user can draw the lines simultaneously. The view is relatively larger than other devices. The connection to an external monitor enables the concurrent sharing of information. One drawback is that this device is slightly more expensive than other similar devices. Detecting venules can be a time-consuming procedure during surgery. Yamamoto et al. reported the use of this system to find veins for LVA [6] or recipient vein for free flap [7]. Vein mapping using this device allowed us to choose the incision points most suitable for LVA. We performed vein mapping after ICG lymphography, just adjacent area from lymphatics, because mapping for the whole leg is very time-consuming and useless. Vein mapping can provide several candidates for LVA incision by crossing of the mapped lymphatics and veins. We could choose the best points among them. All the mapping processes are with no or little invasive procedure, so that we could complete them in the ward or consultation room, and not in the operation room. In the case described here, 7 LVAs were successfully performed at five incision sites. Given the limited surgical time, this time-saving device can lead to more LVAs in the same surgical time. This is important, since the number of LVA is positively correlated with the improvement in 


\section{CASE REPORT}

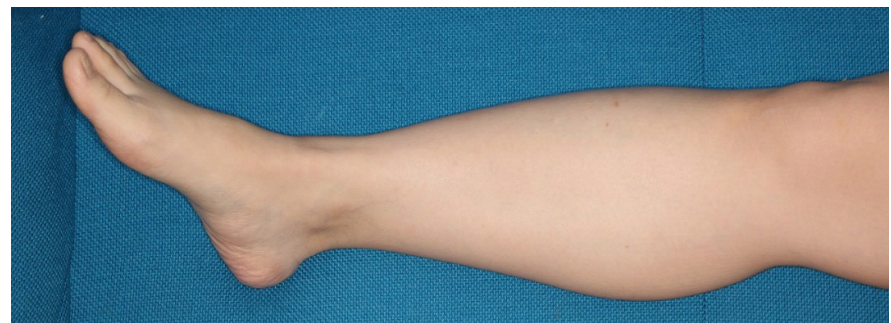

A

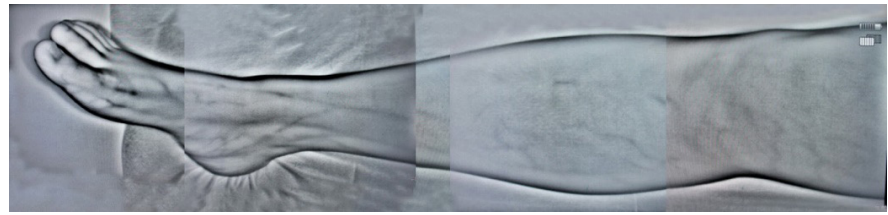

B

Figure 1. (A) Normal view. (B) Veinsite ${ }^{T M}$ view. (C) Veinsite ${ }^{\mathrm{TM}}$ view around the knee. Venules are visible. (D) The red lines were collectors detected by ICG fluorescence lymphography, and the black lines were veins and venules detected by Veinsite ${ }^{\mathrm{TM}}$. The points of intersection between lymphatics and venules were eleven in the right side and nine in the left side. (E) Before operation. (F) Six months after operation.

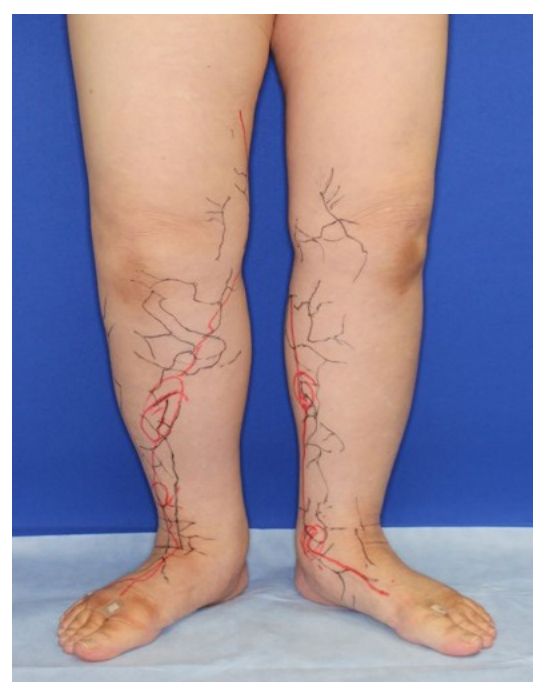

D

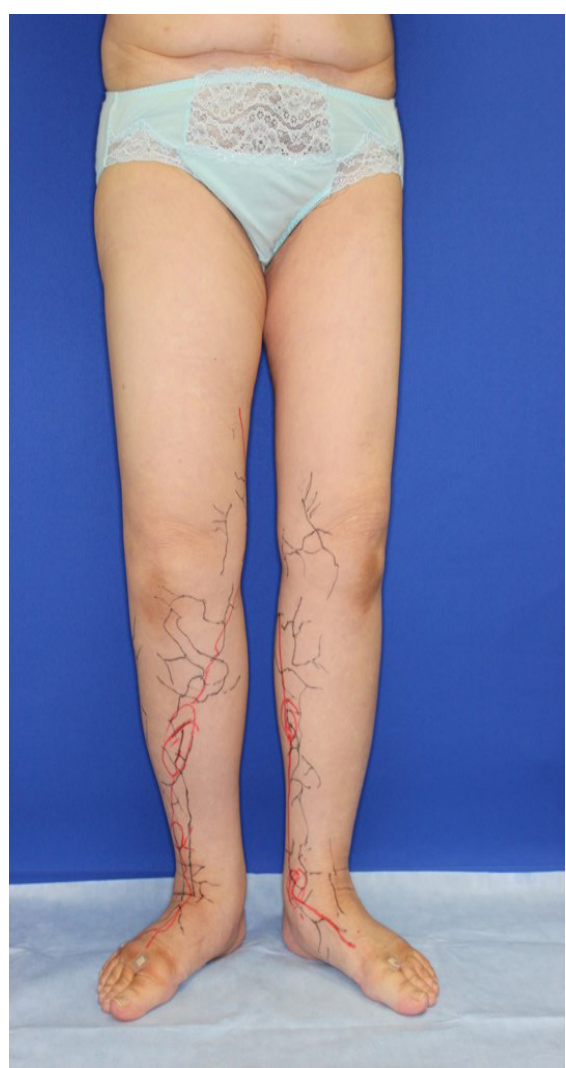

$\mathbf{E}$

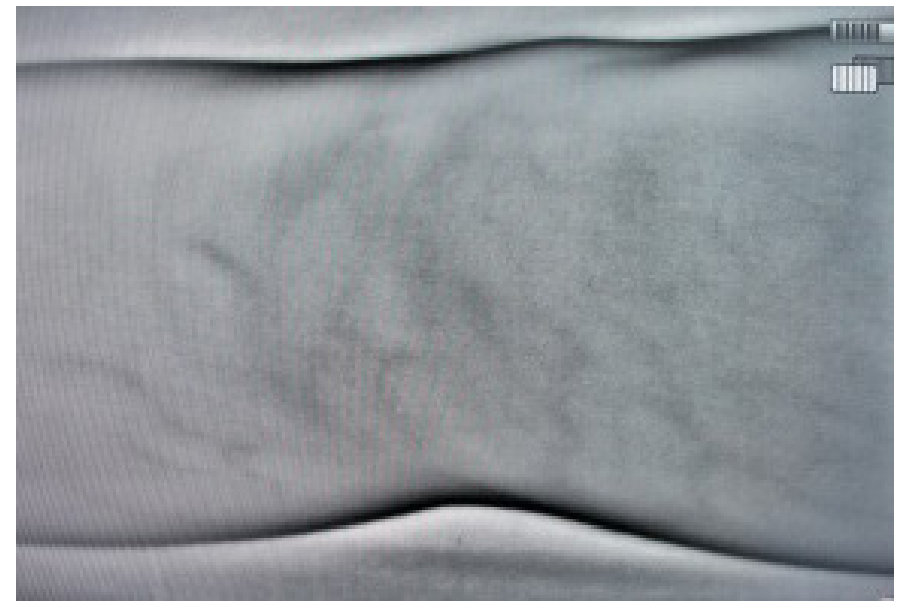

C

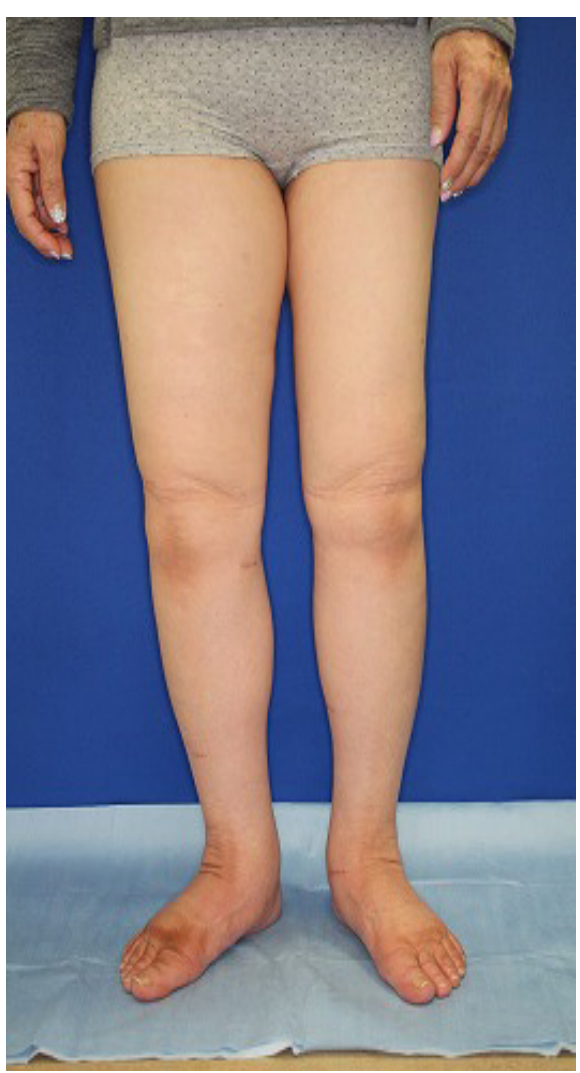

$\mathbf{F}$

lymphedema [1]. The most difficult part of the LVA procedure is in determining where to place the skin incision. Vein mapping can provide additional information to determine LVA site preoperatively, thus increasing the success rate of LVA.

\section{Conclusion}

We reported a case of lower extremity lymphedema treated by LVA that preoperatively mapped not only lymphatic vessels by PDE, but also veins and venules using Veinsite ${ }^{\mathrm{TM}}$. This non-contact vein visualization system could identify venules suitable for LVA, and was helpful for determining the incision site. Preoperative vein mapping can shorten the surgical duration and can increase the anastomoses during the limited time.

\section{Article Information}

*Correspondence: Yukiko Kuramoto, MD

The Cancer Institute Hospital, Japanese Foundation for Cancer Research, Koto, Tokyo, Japan.

Email: yukiko.kuramoto@jfcr.or.jp

This work was presented in the 5th World Symposium for Lymphedema Surgery, Linkou, Taiwan, held on 27-29 April 2016.

Received: Jul. 31, 2017; Accepted: Nov. 20, 2017; Published: Feb. 19, 2018

DOI: $10.24983 /$ scitemed.imj.2018.00053 


\section{CASE REPORT}

Copyright ( $) 2018$ The Author(s). This is an open-access article distributed under the terms of the Creative Commons Attribution 4.0 International License (CC-BY).

\section{Funding: None}

\section{Conflict of Interest Disclosures: None}

\section{Keywords}

Lymphaticovenular anastomosis; lymphedema; vein mapping; Veinsite ${ }^{\mathrm{TM}}$.

\section{References}

1. Ogata F, Azuma R, Kikuchi M, Kikuchi M, Koshima I, Morimoto Y. Novel lymphography using indocyanine green dye for near-infrared fluorescence labeling. Annals of Plastic Surgery 2007;58:652-655.

2. Ogata F, Narushima M, Mihara M, Azuma R, Morimoto Y, Koshima I. Intraoperative lymphography using indocyanine green dye for near-infrared fluorescence labeling in lymphedema. Annals of Plastic Surgery 2007;59:180-184.
3. Yamamoto T, Matsuda N, Todokoro T, et al. Lower extremity lymphedema index: a simple method for severity evaluation of lower extremity lymphedema. Annals of Plastic Surgery 2011; 67:637-640.

4. Mihara M, Murai N, Hayashi $\mathrm{Y}$, et al. Lower limb lymphedema treated with lymphatico-venous anastomosis based on pre- and intraoperative ICG lymphography and non-contact vein visualization: a case report. Microsurgery 2012;32(3):227-230.

5. Mihara M, Hara H, Kikuchi K, et al. Scarless lymphatic venous anastomosis for latent and early-stage lymphedema using indocyanine green lymphography and non-invasive instrument for visualizing subsutaneous vein. Journal of Plastic, Reconstructive \& Aesthetic Surgery 2012;65(11):1551-1558.

6. Yamamoto $T$, Ishiura $R$, Kato M. Hands-free vein visualizer for selection of recipient vein with an intact valve in lymphatic supermicrosurgery. Journal of Plastic, Reconstructive \& Aesthetic Surgery 2015;68(6):871-873.

7. Yamamoto $T$, Ishiura $R$, Hyashi $A$ et al. Hands-free vein visualizer for preoperative assessment of recipient veins. Microsurgery 2016;36(4):351-352. 